\title{
Application of Bioreactor in Stem Cell Culture
}

\author{
Yongxin Zhang ${ }^{1,2}$, Xianghan Wang ${ }^{1}$, Mao Pong ${ }^{1,3}$, Liang Chen ${ }^{4}$, Zhijia Ye ${ }^{3}$ \\ ${ }^{1}$ Nanjing SinoSTEM Engeering Technology Co., Ltd., Nanjing, China; ${ }^{2} Z y x e l l$ Inc., Carrollton, USA; ${ }^{3}$ College of \\ Preventive Medicine, Third Military Medical University, Chongqing, China; ${ }^{4}$ Department of Orthopedic Surgery, \\ Southwest Hospital, Third Military Medical University, Chongqing, China
}

Correspondence to: Zhijia Ye, zjye@tmmu.edu.cn

Keywords: Stem Cells, Bioreactor, Culture, Large-Scale, Biomedical Application

Received: October 17, $2017 \quad$ Accepted: November 14, $2017 \quad$ Published: November 20, 2017

Copyright $\odot 2017$ by authors and Scientific Research Publishing Inc.

This work is licensed under the Creative Commons Attribution International License (CC BY 4.0).

http://creativecommons.org/licenses/by/4.0/

\section{Open Access}

\section{ABSTRACT}

Stem cells (SCs), the undifferentiated biological cells, have the infinite capacity to self-renew and the pluripotent ability to differentiate. SCs and their derived products offer great promise for biomedical applications such as cell therapy, tissue engineering, regenerative medicine and drug screening. However, the clinical applications of SCs require a large amount of SCs with high quality and the number of SCs from their tissue resources is very limited. Large-scale expansion is needed to generate homogeneous SCs with good biological characteristics for clinical application. This necessitates a bioreactor system to provide controllable and stable conditions for stem cell (SC) culture. Traditional methods of bioreactor for maintenance and expansion of cells rely on two-dimensional (2-D) culture techniques, leading to loss self-renewal ability and differentiation capacity upon long-term culture. New approaches for SC expansion with bioreactor employ three-dimensional (3-D) cell growth to mimic their environment in vivo. In this review, we summarize the application of bioreactors in SC culture.

\section{INTRODUCTION}

SCs, a type of cells with infinite proliferation capacity and pluripotent differentiation potential, can roughly be categorized as embryonic stem cells (ESCs), adult stem cells (ASCs) and induced pluripotent stem cells (iPSCs). SCs can be characterized by their self-renewal capability and pluripotent differentiation ability. Specifically, a SC can proliferate via self-renew or differentiate into other cell types under certain conditions. The fate of SCs is controlled by its biological characteristics and external environment.

With the development of basic researches and technologies related to SCs, SC transplantation and SC-based tissue engineering provide the possibilities for the treatment of some refractory diseases. In clinical practice, SCs are mainly applied in three fields: 1) SCs transplantation: ASCs can be transplanted into 
patients by intravenous infusion or local injection for therapeutic uses. With the emergence and development of gene editing technology, the mutated gene in the SCs of patients can be corrected and then the edited SCs are transplanted to replace the mutated SCs in these patients, which represents a direction in SC treatment $[1,2] .2)$ Construction of tissues or organs: SCs can be used as seed cells and cultured with tissue compatibility biomaterials to construct tissues or organs which are then used to repair the lesion of tissues or organs $[3,4] .3)$ Toxicology and drug screening: SCs provide a basis for the studies on the toxicology and pharmacokinetics of a new drug at the cell level, which reduces the requirement for animal test or clinical test [5]. This indicates that there are a great demand for SCs in the clinical cell transplantation, regenerative medicine and drug screening, and SCs have presented great prospects in the biomedical science.

However, the clinical application of SCs encounters an important challenge. The lack of clinically eligible SCs is a critical problem that limits the wide clinical application of SCs. The initial number of SCs obtained from their sources is not sufficient for treating patients. For example, the SCs including hematopoietic stem cells (HSCs) and mesenchaymal stem cells (MSCs) from umbilical cord blood and umbilical cord tissue have a low immunogeneticity and a high self-renewal capability and high differentiation ability, but these primitive SCs are quantitatively insufficient for the clinical application. ESCs or iPSCs may serve as important sources of ASCs such as neural stem cells (NSCs) and myocardial stem cells that cannot be obtained from tissues, but the induction efficiency and separation efficiency of the target stem cells from ESCs or iPSCs remain very low. The large-scale expansion of ESCs or induced ASCs is needed before its application. Thus, large scale expansion of SCs in vitro is an important way to provide sufficient SCs for clinical application.

Compared to the differentiated cells, SCs have three ways for the mitosis: Symmetrical self-renewal, symmetrical differentiation and asymmetrical self-renewal and differentiation. The fate of SC mitosis is mainly dependent on the niche in vivo [6,7]. With the implementation of in depth investigations on the components in the niche, the ways to act and the regulatory network as well as the mechanisms underlying the interaction between niche and SCs, it is feasible to artificially construct a niche to regulate the fate of SC mitosis and change the fate of SCs [8]. Besides the components of the niche, the spatial structure of the niche is also important to regulate the fate of SC mitosis [7]. The SCs, especially HSCs, are sensitive to structure of the niche, such as the structure of bone marrow, with its unique anatomic space, is favorable for the self-renewal of adult HSCs. Since in vitro expansion of SCs requires maximizing the symmetrical division of SCs and minimizing their differentiation, it would be necessary to mimic the in vivo components and spatial structure of the niche during SCs in vitro expansion. Currently, variable bioreactors have been constructed to mimic SC niche in vitro. A typical bioreactor includes three functional parts (cell chamber, monitor and controller) and can provide controllable and stable environment for the SC culture. A bioreactor-based system can be automated to perform the necessary medium exchanges and reduce culture variations and microbial contamination by avoiding the daily culture maintenance. Cells in bioreactors can be cultured in either static or dynamic state. Conventional static culture is 2-D-based, has several drawbacks such as critical concentration gradients of $\mathrm{pH}$ and dissolved oxygen and metabolites. Moreover, the proliferation of SCs is significantly affected by their spatial distribution. In contrast, dynamic culture can provide cells 3-D growth environment, and cells in dynamic culture can receive adequate metabolic support but are often negatively affected by the sheer-stress during the cell suspension. Currently, there are several types of bioreactors commercially available for SC culture. Although it is difficult to compare the performance of different bioreactors in SC culture based on expansion rates and/or achievable cell densities, accumulating data from available studies using different bioreactors indicate that the development of bioreactors is very important step for the expansion of different SCs including the suspension and adherent SCs $[9,10]$. Novel systems and approaches, therefore, are still needed to offer suitable strategies to expand SCs in vitro. Bioreactors such as stirred tank [11, 12], wave-rocking [13, 14], rotating wall vessel $[15,16]$ and hollow fiber bioreactors $[17,18]$ have been recently studied for SC expansion. Herein we summarize these bioreactors widely used for expansion of SCs according to their rationality, characteristics and mode in SC culture. 


\section{BIOREACTORS USED FOR SC EXPANSION}

In the SC culture, bioreactor often plays critical roles. It can provide suitable environment for SC culture and determine the quality and quantity of cultured SCs. According to the requirements for SC growth, the bioreactors should have low shear stress, good transferring effect and good hydrodynamics. To date, several types of bioreactors have been developed for the SC expansion.

\subsection{Stirred Tank Bioreactor}

Stirred tank bioreactor is the first and the classic bioreactor. The twirling of the stirrer may assure the uniform distribution of cells and nutrients, and thus the cells can make full use of these nutrients, increase the gas-liquid interface, provide sufficient oxygen for cell growth and maintain stem cells in normal metabolism.

This system has relatively simple internal structure, and is applicable for both suspension and adherent cell culture. Several stirrers have been improved based on the different stirring styles, including BIOSTAT B bioreactor (Sartorius, Germany) with two blade impellers and bubble-free aeration system and CelliGen bioreactor (New Brunswick Scientific) with Cell-lift double-screen Cell-Lift impeller. In addition, EMD Millipore worked with the Centre for Commercialization of Regenerative Medicine (CCRM) to develop the optimized stem cell culture system based on the Mobius Cell Ready stirred tank bioreactor.

Stirring bioreactors were used for expansion of different types of SCs including suspension and adherent SCs. Suspension SCs like hematopoietic progenitor cells without requirement of surface attachment for growth were successfully cultured in stirring bioreactors. Adherent SCs, such as ESCs, iPSCs and MSCs, are attached to cell microcarriers for their suspension and are cultured in stirring bioreactors [19, 20]. The growth efficiency and homogeneous of cultured SCs in stirring bioreactor were improved in somehow, due to homogeneous nature of culture system. However, SCs are sensitive to shear stress, and agitation as well as aeration affects the cell behaviors such as proliferation and differentiation [11, 21].

Although stirring bioreactors which are relatively simple and readily scalable in providing a homogeneous environment and are easy to operate, allowing sampling, monitoring and control of culture conditions, its inherited shear force may result in damage to stem cells and induction of non-specific differentiation during the SC expansion.

\subsection{Wave-Rocking Bioreactor}

Wave-rocking bioreactor is also known as Cellbags bioreactor. In this bioreactor, cells and medium are placed in a sealed, sterilized airtight plastic bag, which is then placed on a rocking platform. The wave motion of the medium in the bag along with the rocking of the platform may achieve a favorable mixing of cells and medium.

This reactor was first commercialized by GE company, and its brand name is Wave Bioreactor [14]. The German Sartorius company also developed a similar bioreactor named BIOSTAT RM.

The advantage of this system is that the moderate wave motion may achieve favorable oxygenation and mixing effect. In addition, the wave motion may produce a shear force smaller than that produced by stirring or air-lifting. The undulating medium may provide enough dissolved oxygen for cell growth. The frequency and angle of platform rocking can be adjusted, and the air/oxygen at a specific ratio can be infused into the bag, which is helpful for the intentional increase of dissolved oxygen concentration.

This system is applicable in the culture of different cell types, including CHO cells, hybridoma cells, lymphocytes, plant cells and some cell lines, but SCs are seldom cultured with this system because the ceaseless wave-like motion during the cell mixing still produces the great shear force and causes cumulative damage to SCs [22].

\subsection{Rotating Wall Vessel Bioreactor}

Rotating wall vessel bioreactor (RWV) is a spatial microgravity culture system and employs rotating 
vessels for cell culture. In this system, the medium and cells or tissues are added to a culture vessel and the bubbles in the medium are removed. During the rotation of the culture vessel, the tissues, cells or cell clumps remain in a suspension status due to a balance between rotational centrifugal force and cell gravity. Rotation may allow the even exchange of nutrients and gas among cells and there is extensive contact between cells during the rotation. There is a membrane oxygenator at the dorsal side of the culture vessel for gas exchange, which allows the sufficient oxygenation and expelling of metabolites.

One of the advantages of this system is the suspension status of cells or tissues in the medium [16, [23], which avoids the damage produced by the impellers or air bubbles to SCs. In addition, the force experienced by cells at different directions is balanced in the container and thus cells may not be affected by the force at a single direction. This may achieve even spatial growth, reduce cell damage and effectively maintain SC function. To date, this system has been successfully used for the expansion of different types of SCs and tissue culture $[16,24]$.

However, there is still cumulative stress produced during vessel rotation in RWV system, which may lead to the loss of self-renewal ability of SCs [15]. Moreover, the long term microgravity may alter cytoskeleton structure and gene expression pattern in SCs [24, 25].

\subsection{Hollow Fiber Bioreactor}

In the hollow fiber bioreactor, the culture chamber consists of thousands of hollow fibers, and each hollow fiber is perfused with culture medium for cell growth, the space between hollow fibers is known as external chamber, and cells are adherent to the external wall of hollow fibers and can take nutrients from the inner chamber. The metabolites produced during cell growth can enter the inner chamber via the wall, are finally expelled from the general export and may not cause damage to cells in the external chamber.

This system has a moderate culture niche which has little damage to cells. In addition, the cell density is very high, and thus cells are easy to separate [26]. This system has been successfully applied in the large-scale expansion of MSCs [27].

However, this system still employs 2-D culture and is unable to provide sufficient spatial and metabolic support for SC culture. Currently, it is mainly applied in the culture of MSCs [26, 28].

\subsection{Multi-Plate Bioreactor}

The Xpansion bioreactor is made of stacked circular plates which increase the surface of cell growth and the area for gas-liquid exchange. The medium and gas entering the bioreactor via the perfusion system provide metabolic support for the high-density cell growth, and thus 2-D large-scale culture of adherent cells is feasible.

This bioreactor provides a sufficient surface for the large-scale culture of adherent cells, and the compact design between plates abolishes the gas phase between plates. The central column at the center of the plate allows the circulation of culture mediator in the bioreactor and the gas exchange, which provides metabolic support for cell culture. For the cell collection, the rotation of this system may produce mechanical force for cell separation. The system has been successfully used for large-scale expansion of MSCs [29].

This system still employs 2-D culture and is hard to assure the even distribution of cells during culture and perfusion.

\subsection{Magnetic Microcarrier Bioreactor}

BioLevitator bioreactor employs magnetic Global Eukaryotic Microcarrier (GEM) and automated program control and mimics 3-D environment for cell culture. It is used for high density suspension culture of adherent SCs and can maintain the biological characteristics of expanded cells. This provides a precision cell model for drug screening.

BioLevitator 3-D cell culture system composes of a desktop incubator and a bioreactor, and can be used for the independent high density cell culture in quadruplicate. This system does not need accessory 
equipments such as cell incubators and centrifuges, and minimizes the manual manipulation. The magnetic GEM developed by the Global Cell Solutions employs microcarrier cell culture for 3-D culture. The microcarriers are the microspheres with 3-D curved surface sized $75-150 \mu \mathrm{m}$ in diameter. These microspheres are composed of compressible, porous alginate and pre-coated with different types of extracellular matrix (ECM) which provide a favorable environment for the culture of different cell types. The magnetic core in the microcarrier makes the medium change and cell collection convenient and simplifies the manipulation [30]. This bioreactor with 3-D culture environment produces small shear force and thus is applicable for the culture of adherent SCs [20].

However, this system is small and cannot be applied in large-scale expansion of cells. In addition, it is inapplicable for the culture of suspension cells.

\subsection{D Scaffold Bioreactor}

The Z PR bioreactor (Zellwerk GmbH) employs cell culture chamber in which there are Sponceram scaffolds made of porous materials. Cells grow on the scaffolds, the culture chamber rotates around the horizontal axis and the scaffolds are alternatively immersed in the medium for nutrient exchange [31]. The moderate rotation may stimulate the adhesion and proliferation of cells and tissues without the influence of shear force [32]. The specific rotation program combines with the solution for cell separation to facilitate cell collection.

The Cocoon bioreactor developed by the Octane Company is a closed culture system and applicable for individualized therapy. Octane Company developed a novel cell scaffold and culture box [33]. The scaffolds are placed in the culture box, and cells are cultured in this sealed box. This bioreactor is applicable for the culture of tissues and organs, but inapplicable for the large-scale expansion of SCs.

\subsection{Microfluidics Lab on Chip}

The microfluidics lab on chip integrates the components for the sample preparation, reaction, separation, detection and control into a microarray sized several square centimeters in which microchannels forms a network to control the microfluidics in the whole system. Thus, the functions of common labs are replaced by this system, and-this chip has the characteristics of high throughput, integration and small reagent consumption.

The components in the microfluidics lab on chip are generally composed of cell culture unit and fluid control unit. The cell culture unit is used for cell culture, sorting and detection; the fluid control unit is composed of micropump and microvalve and designed for the control of microfluidics flow among cells. The CellASIC ${ }^{\mathrm{TM}}$ microfluidics lab on chip developed by Merck Millipore mainly consists of fluid controller, temperature and gas controller, multi-control panel, chip culture plate and FG control software. The flow of microfluidics is driven by the gravity for cell seeding and medium exchange. That is, cells or medium is added through the inlet until the medium surface is higher than the height of the outlet. In the presence of gravity, medium flows into the microchannels of the chip, which is the precise, dynamic "Lab-on-Chip" of cells $[34,35]$. The medium at the outlet and inlet reaches the same level, and thereafter the medium flow begins to stop. Thus, it is hard to maintain the flow of medium in the microchannels.

One of the advantages of this system is that the miniaturized cell operating system reduces the consumption of reagents, which not only decreases the cost, but is convenient for the coupling of different components. This system is especially applicable in studies on the relationship between cells and microenvironment [36]. The medium can be precisely controlled at the microscale level with this system. In addition, this system can combine with microscope to automatically monitor cell growth for a long time and to dynamically detect the cell growth in a real time manner by setting the operating software [37]. In the co-culture of human embryonic stem cells (hESC), PA6 stromal cells and separated nerve plexus structure, neurospheres can form in the medium [38]. The microfluidics control technique can be employed to monitor the differentiation and migration of neurons derived from hESC [39]. However, this system has a small culture volume and is inapplicable for large-scale expansion of SCs. 


\subsection{Kinetic and Static Alternating Bioreactor}

Kinetic and static alternating bioreactor can mimic the dynamic in vivo microenvironment of human cells and is applicable for the high efficient expansion of sensitive cells (such as HSCs and immune cells) with the reduced shear force, which assures the cell characteristics, especially the stemness of SCs [40, 41]. This system is composed of inversion device, cell culture chamber and control system. The inversion device includes the inversion scaffold and magnetic field generating device. The inversion scaffold can rotate around the space axis and the magnetic field generating device is physically connected to the inversion scaffold. The cell culture chamber can be fixed on the inversion scaffold at the space axis or its extension line and may rotate along with the inversion scaffold following the designed movement trajectory. The cell culture chamber is close to the magnetic field generating device which may control the peripheral and inner magnetic field of the chamber.

This bioreactor has two types: magnetic intermittent cell suspension and intermittent vertical suspension. In the magnetic intermittent cell suspension, the magnetron carriers may stir up and down evenly, which assures the even distribution of cells after the culture changes from kinetic to static. In the intermittent vertical suspension, the culture chamber rotates vertically for cell suspension, and changes into static culture at horizontal level controlled by its deceleration program for the even distribution of cells in the chamber. In both types of bioreactors, the kinetic and static cultures are controllable, and the combination of kinetic and static cultures minimizes the shear force on cells during the culture; cells display even distribution in the transformation between kinetic and static cultures. The kinetic and static states are controlled based on the cell growth state. The kinetic state aims to improve the nutrient supplement and cell metabolism due to their even distribution; the static state provides a favorable microenvironment. The kinetic-static alternative cell culture may achieve favorable medium stirring in a moderate, gentle and intermittent manner, so that limits the shear-stress exerted on cells. This further increases the environmental stability for cell culture and the even distribution of cells, which minimizes the non-specific differentiation of SCs and the damage induced by mechanical stirring to SCs. Moreover, this system is able to control the suspension of cells and carriers during the stirring, which is better to create a microenvironment for cell growth and metabolic support.

In recent years, studies on the kinetic and static alternating bioreactor have shown the advantages of this system: 1) the computer-controlled vertical suspension system allows the magnetic agitator to combine buoyancy and gravity to vertically suspend cells; 2) in the same cell culture chamber, cell culture can be in static state, kinetic state and kinetic-static alternative state. The static culture after kinetic state may achieve even distribution of cells, which minimizes the shear force and achieves a favorable environment for cell culture. 3) This system is able to automatically sort cells and dynamically monitor the culture environment in a real time manner. That is, this system allows the cell sorting and cell culture in the same chamber, which significantly reduces the cell loss and avoids the potential contamination during the cell sorting and transferring. This kinetic-static alternating bioreactor has been applied in the culture of hematopoietic stem cells and immune cells and has achieved promising findings. In the expansion of hematopoietic stem cells, this system reduces the non-specific differentiation, provides an effective metabolic support for cell growth and displays a lot of advantages over other systems [41]. There is evidence showing that the use of kinetic-static alternating bioreactor significantly increases the expansion efficiency of cytotoxic T lymphocytes (CTL), and CTL after expansion show highly specific cytotoxicity to cancer cells in vivo and in vitro. However, this system has not been applied in clinical trial.

Bioreactors that could be used for SC expansion are listed in Table 1.

\section{PARAMETERS AFFECTING SC EXPANSION IN BIOREACTORS}

The key parameters in the SC culture with bioreactors include dissolved oxygen (DO), fluid shear stress, growth factors, nutrients and metabolism. Shear stress is an important parameter for the design of bioreactor. SCs are sensitive to shear force, and a larger shear force may cause to damage to SCs and affect their differentiation. These key parameters should be optimized and rationally monitored, aiming to provide 


\section{Table 1. Bioreactors used for SC expansion.}

\begin{tabular}{|c|c|c|}
\hline Type & Products and Manufacturers & Reference \\
\hline Stirred tank & $\begin{array}{l}\text { BIOSTAT B bioreactor (Sartorius, Germany)/Mobius } \\
\text { Cell Ready bioreactor (EMD Millipore, } \\
\text { Germany)/CelliGen bioreactor } \\
\text { (New Brunswick Scientific, USA) }\end{array}$ & {$[12,13,20,21]$} \\
\hline Wave-rocking & $\begin{array}{l}\text { Wave bioreactor (GE, USA)/BIOSTAT RM } \\
\text { bioreactor (Sartorius, Germany) }\end{array}$ & {$[14,15]$} \\
\hline Rotating wall vessel & RWV bioreactor (Synthecon, USA) & {$[16,17,24,25]$} \\
\hline Hollow fiber & $\begin{array}{c}\text { Quantum CES bioreactor } \\
\text { (Terumo BCT, Japan)/FiberCell } \\
\text { hollow fiber bioreactor (USA)/CellMax } \\
\text { hollow fiber bioreactor (Spectrum, USA) }\end{array}$ & {$[18,19,27,28]$} \\
\hline Multiplate & $\begin{array}{c}\text { Xpansion bioreactor (Pall, USA)/CellCube } \\
\text { bioreactor (Corning, USA) }\end{array}$ & {$[30]$} \\
\hline Magnetic Microcarriers & $\begin{array}{l}\text { BioLevitator bioreactor and Global Eukaryotic } \\
\text { Microcarrier developed by Hamilton } \\
\text { (Switzerland) and Global Cell Solutions (USA) }\end{array}$ & {$[21,31]$} \\
\hline 3D scaffold & $\begin{array}{c}\text { Z PRbioreactor (Zellwerk GmbH, Germany)/Cocoon } \\
\text { bioreactor (Octane, Canada) }\end{array}$ & {$[33,34]$} \\
\hline Microfluidics lab on chip & $\begin{array}{l}\text { CellASIC }{ }^{\mathrm{TM}} \text { microfluidics lab on chip } \\
\text { (Merck Millipore, USA) }\end{array}$ & {$[38-40]$} \\
\hline $\begin{array}{l}\text { Kinetic-static alternating } \\
\text { bioreactor }\end{array}$ & $\begin{array}{c}\text { ZYX bioreactor (Zyxell, USA)/SinoSTEM } \\
\text { bioreactor for stem cells (Nanjing SinoSTEM } \\
\text { Engeering Technology Co., Ltd) }\end{array}$ & {$[41,42]$} \\
\hline
\end{tabular}

the optimal conditions for SC expansion. Ideally, all parameters should be automatically measured by the bioreactor system. However, some parameters are especially important for a certain stem cells. For example, oxygen concentration and fluid shear stress are extremely important for maintaining the self-renewal capacity of HSC, and cell carriers are the basic supporting materials for MHC growth.

\subsection{Oxygen Concentration}

Oxygen is one of important factors affecting the metabolism of tissues and cells in physiological niche and a crucial regulator of cell physiology [42]. Generally, animal cells are maintained at constant $37^{\circ} \mathrm{C}$ in a humidified environment with $5 \%-10 \% \mathrm{CO}_{2}$. In the standard $\mathrm{CO}_{2}$ incubator, oxygen fraction is a key parameter of gas phase in the cell culture. The gas with normal oxygen fraction is also known as normoxia; the gas with lower and higher oxygen fractions is also known as hypoxia and hyperoxia, respectively. Oxygen concentration is an important component of the niche in the in vitro SC expansion, and also crucial 
for the proliferation and differentiation of SCs and the stemness maintenance $[42,43]$.

HSCs and precursor cells are distributed in the bone marrow along the oxygen gradient, HSCs survive in the area with the lowest oxygen concentration, and hypoxia is beneficial for the HSC activity [44]. A variety of studies have been conducted to investigate the influence of oxygen concentration on the function of HSCs. HSCs are distributed in the bone marrow and umbilical cord blood which possess a hypoxic environment, but the separation and culture of HSCs are usually performed in non-physiological conditions $[44,45]$. The exposure of bone marrow and umbilical cord blood to normoxia may induce the differentiation of HSC, and reduce the efficiency of HSC expansion, which is also known as extraphysiologic oxygen shock/stress (EPHOSS). In the harvest of HSCs, the addition of cyclosporine A may protect HSCs against EPHOSS, which increases the proliferation capability and transplantation efficiency of HSCs [45].

Oxygen concentration can regulate the proliferation and differentiation of MSCs and affect the osteogenic, chondrogenic and adipogenic capabilities of MSCs [43, 46]. Dos Santos et al cultured hMSCs with $2 \%$ oxygen, and they found that $2 \%$ oxygen was better to promote the growth and expansion of MSCs as compared to normoxia. hMSCs could be passaged 7 times in a hypoxic environment ( $\%$ oxygen), and the fold of expansion was as high as 30 within 6 weeks without loss of differentiation potential. Adipose derived MSCs (ADMSCs) were maintained in normoxic $\left(21 \% \mathrm{O}_{2}\right)$ or hypoxic $\left(1 \% \mathrm{O}_{2}\right)$ environment, and results showed hypoxia was beneficial for the proliferation and the maintenance of their stemness, which enriches the cells with differentiation potential and makes the long term expansion possible [47].

In the large-scale expansion of SCs with bioreactors, the gas concentration, the way and rate of ventilation, and the gas flow can be controlled to regulate the oxygen supply, creating an environment with optimal oxygen concentration for SC expansion.

\subsection{Fluid Shear Force}

In vitro expansion and culture of SCs can employ dynamic or static mode. In the dynamic culture of SCs, bioreactors are used. The fluid in the bioreactor may produce fluid shear force, which may affect the expansion of SCs and their physiological functions [23]. Small shear force may increase the cell permeability, leading to the increases in the nutrient uptake, proliferation activity and the fold of expansion. The increasing shear force may inhibit the SC proliferation, and promote their differentiation, or even cause damage to SCs, which especially may result in loss of proliferation potential and hematopoietic activity of HSCs [15].

SCs are sensitive to the shear force produced by stirring. In the stirring tank bioreactor, the energy may transfer from the impeller to the medium, leading to an intense movement of the medium, which then alters the receptors of growth factors on cells and affects the expansion efficiency [25]. The maximal shear force SC can bear varies among different types of SCs [22].

\subsection{Nutrient Supply and Metabolism}

Cell survival and growth are largely dependent on local environments and there exists continuous exchange between cells and surrounding environment: cells get nutrients from the surrounding environment and release metabolites. Nutrients (such as glucose and amino acids) may provide essential energy for the cell growth. Some toxic metabolites (such as lactic acid, ammonia and reactive oxygen species) may influence the environment for cell culture (such as $\mathrm{pH}$ and osmotic pressure), leading to the inhibited cell growth and reduced cell viability. Fernandes et al. investigated the matrix consumption and production of by-products in the expansion of mESCs in serum free medium, and they found mESCs had a high metabolic level and uncovered that glutamate was an important source of energy for the cell proliferation [48].

In recent years, power plants are implanted into bioreactors, and the employment of different sensors makes the continuous monitoring and adjustment of parameters related to cell growth possible, which lead to the generation of an optimal environment for cell growth [49]. Dynamic perfusion culture allows the addition of fresh medium and retraction of metabolites of cells, which assures the culture of cells at a high density, generating a vigorous growth status [50]. This culture is also helpful for the reduction of cell 
contamination.

The limitation of animal serum extraction causes difficulties in the cell culture such as standardization of cell culture, the separation and purification of products, the potential mycoplasma contamination, the lack of serum supply and the increased cost. Thus, serum free culture is a direction in the future standardized production of biological products with bioreactors [51, 52]. It is necessary to investigate the metabolic characteristics of SCs in large scale expansion of cells, design the key components in the serum free medium and optimize the physical and chemical properties of the medium, which may develop individualized culture medium [53]. This, together with appropriate fed-batch or perfusion strategies, may assure the key nutrients at appropriate levels, which reduces the production of toxins and is suitable for large-scale cell expansion [10,54].

\subsection{Microcarriers}

Microcarriers, small beads sized $100-300 \mu \mathrm{M}$ in diameter, are synthesized with various materials, including agrose, polystyrene, gelatin, dextran, and collagen. They provide an adhesive surface for anchorage-dependent cells in suspension culture. Microcarriers also provide a larger surface area for adherent cells to grow at higher densities $[10,50,55]$.

Dynamic culture techniques combining microcarriers with spinner flasks have been shown to support human ESC and MSC proliferation to high densities [56, 57]. There are several types of microcarrier commercially available. Optional combinations of different bioreactors with different microcarriers have been successfully applied in the expansion of SCs. For example, human ESCs adherent to microcarriers cultured in continuous perfusion RWV bioreactor system under xeno-free conditions without animal derived materials resulted in more than 10-fold increase in the growth, and more than 100-fold increase in cell number was achieved for human MSCs adherent to microcarrier cultured in Stirred bioreactor.

The surface of microcarrier can be coated with different biomaterials such as vitronectin, laminin or Matrigel [22, 57]. The biomaterials coated on microcarrier surface mimic characters of ECM or 3-D structure of the niche to promote the proliferation or differentiation of SCs. Cellulose microcarriers coated with an assortment of ECM proteins have been used for long-term expansion and maintenance of human ESCs. Microcarriers coated with ECM proteins such as Matrigel have been shown to promote pluripotent SC growth.

Another main advantage of dynamic culture using microcarriers allows subsequent differentiation into specific cell lineages after expansion. Microcarriers have been demonstrated to support the differentiation of EBs into hemangioblasts, which are capable of differentiating into hematopoietic and endothelial cells $[58,59]$.

Microcarriers were also used to generate tissue-like structures in the cell culture. This kind of structure lacks blood supply system for the metabolic support to the cells in its central parts, which could result in "central necrosis" as occurred in cancer mass. In addition, the formation of this structure with microcrriers is almost inevitable in all kind of cell culture and it is a defect for HSC expansion since the HSCs in the formed "tissue", similar to the cell clump, are often damaged, non-specifically differentiated and hard to be harvested.

The SC expansion in vitro is influenced by multiple factors. However, a factor may not act alone and it usually functions with other factors to affect the niche for cell culture, which then influences the growth and biological function of SCs. Thus, many factors should be taken into account in the design and material selection of bioreactors to optimize the culture conditions of SCs.

\section{CHALLENGES}

Great progress has been made in understanding the mechanisms of self-renewal, pluripotency, and differentiation of SCs, and new xeno-free media have been developed to culture SCs that can be used for clinical purposes. However, little progress has been achieved regarding the large scale expansion of SCs such as HSCs and progenitor cells. Moreover, the clinical eligible expansion of ESCs and MSCs still needs 
to be further evaluated and standardized.

\subsection{Biological Function}

The major consideration of SC culture is that the cultured cells should remain same cell surface markers and functional activity in vitro as the original uncultured SCs. Most importantly, the cultured SCs should also be able to survive and integrate into the tissues in vivo after transplantation. The critical parameters including chemical and physical factors affecting the functions of cultured SCs should be considered prior to their use for clinical purposes. Furthermore, the development of efficient approaches to yield homogenous SCs may help to yield reproducible results. Future studies should focus on the investigation of temporal factors in the in vivo conditions and construction of microenvironments using appropriate biomaterials for the growth and differentiation of SCs.

\subsection{Optimization of Culture Conditions}

Although a great progress has been achieved in the growth and differentiation of SCs using bioreactor, the standardized procedures for SC culture are still lacking. 2-D culture methods are simple and easy, but less efficient and less reproducible. A 3-D culture system involves a number of parameters, including mechanical stress, $\mathrm{pH}$ control, medium perfusion and biomaterials, and the optimization of these parameters is beneficial for the improvement of both quality and quantity of cultured SCs. The control programs of a bioreactor for the nutrient diffusion/waste exchange are also important for the standardization of SC culture. The recent advances in the use of various bioreactors to expand different types of SCs are promising $[9,60]$. Dynamic bioreactors have shown many advantages over static bioreactors. However, dynamic bioreactors employing agitation or media perfusion could introduce hydrodynamic shear stress, which usually have negative effects on cell viability and increase nonspecific differentiation. The kinetic-static alternating bioreactor definitely overwhelms other bioreactors in balancing between shear stress and metabolic support for maximizing the cell expansion efficiency. When kinetic-static alternating bioreactor is used in cell expansion, the ratio of kinetic/static is very important for the expansion efficiency. Recent published studies demonstrated that excessive kinetic with a higher kinetic/static ratio could result in the apoptosis of the cells in the culture [40,61]. Therefore, the optimization of kinetic/static ratio in cell expansion with kinetic-static alternating bioreactor should not be ignored.

\subsection{Biomaterials}

3-D culture of SCs usually employs biomaterials to mimic the biochemical and biophysical properties of the 3-D niche in vivo. Numerous natural and synthetic biomaterials have been used to develop suitable scaffolds to mimic in vivo environment for cell growth $[20,62,63]$. However, it is often difficult to determine which biomaterial would be most appropriate for the growth and differentiation of a particular SC type. For instance, different biomaterials induce variability and reduce reproducibility when used in SC culture. Biomaterials are also known to play role in signaling and may also affect the maintenance and differentiation of SCs. Therefore, further studies are needed to identify appropriate biomaterials that can improve the quality and quantity of cultured cells.

\subsection{Culture Scale Up}

A large amount of high-quality SCs is needed for their biomedical applications. The HSCs without expansion normally have limited cell number and it may be enough for the autologous transplantation in individual patients. However, allogous SC therapy for multicenter clinical trials requires billions to trillions of cells. Scale-up expansion of SCs to such levels requires substantial advancements in the medium, culture devices and techniques. Bioreactors and GMP facilities are necessary for large-scale production systems. In addition, a combination of techniques, including biomaterials modified with growth factors or ligands as well as other growth parameters to optimally grow SCs will further advance the fields of cell 
therapy, tissue engineering and regenerative medicine.

\section{EVALUATION OF CELL EXPANSION EFFICIENCY}

Unlike other cells which expansion efficiency can be evaluated simply by cell counting, some SCs lose their self-renewal capacity and differentiate to other cells during expansion. These differentiated cells are no longer SC and should be excluded when the cell expansion efficiency is evaluated. A typical example is the HSC expansion. When HSCs are cultured in vitro, cells increase size and granularity and gain CD33 and may lose CD34 and CD 133 [64]. The cells which become positive for CD33 or/and have significant greater size and more granular should not be counted as effectively expanded HSC, no matter if they are positive for CD34 and CD133 or not. However, many researchers are still only use CD34 or/and CD133 to evaluate the HSC expansion efficiency. The studies without excluding differentiated cells in their results definitely overrated their expansion methods and might be misleading. Since the evaluation methods for SC expansion in different studies lack consistency, it is very difficult to compare their expansion efficiency. Therefore, it is also necessary to standardize the evaluation methods of SC expansion. For HSC expansion, it was suggested to the use the protocol developed by the International Society of Hematotherapy and Graft Engineering (ISHAGE) [65] to numerate the HSC with flow cytometry because it has been most broadly applied in clinical HSC counting.

\section{CONCLUSION}

The goals of SC expansion in vitro for biomedical application mainly are 1) to obtain sufficient SCs for autologous transplantation, 2) to maintain biological characteristics of SCs to the utmost for long-term engraftment, 3) to establish reproducible procedures to obtain stable and homogeneous SCs, and 4) to be cost effective. Bioreactor and biomaterial are essential to reach those goals. The studies on the SC culture using bioreactors and biomaterials have shown significant advantages in SC expansion for clinical application. The 3-D culture of SCs has a great potential to expand SCs for clinical application without loss of long-term engagement ability. The combination of 3-D culture of cells with biomaterials mimicking the ECM, dimensionality and spatial gradients in the SC niche in vivo may be helpful for the SC expansion and the improvement of functions of cultured SCs. Scale-up expansion of SCs with bioreactors and GMP facilities may benefit from the collaborations among biomedical researchers and bioreactor engineers. Despite challenges, the understanding of the mechanisms under the regulation of SC fate will guide improvement of the SC expansion using bioreactor. The advances in 3-D culture systems will accelerate the clinical application of SCs in the treatment of many diseases and disorders that have no curative modalities.

\section{ACKNOWLEDGEMENTS}

This research was supported by NSFC $(81170471,31371393)$ and R\&D program of Chongqing (cstc2014yykfa110005).

\section{REFERENCES}

1. Hoban, M.D. and Bauer, D.E. (2016) A Genome Editing Primer for the Hematologist. Blood, 127, 2525-2535. https://doi.org/10.1182/blood-2016-01-678151

2. Vassena, R., Heindryckx, B., Peco, R., et al. (2016) Genome Engineering through CRISPR/Cas9 Technology in the Human Germline and Pluripotent Stem Cells. Human Reproduction Update, 22, 411-419. https://doi.org/10.1093/humupd/dmw005

3. Lancaster, M.A., Renner, M., Martin, C.A., et al. (2013) Cerebral Organoids Model Human Brain Development and Microcephaly. Nature, 501, 373-379. https://doi.org/10.1038/nature12517

4. Sun, Y., Yong, K.M., Villa-Diaz, L.G., et al. (2014) Hippo/YAP-Mediated Rigidity-Dependent Motor Neuron 
Differentiation of Human Pluripotent Stem Cells. Nature Materials, 13, 599-604.

https://doi.org/10.1038/nmat3945

5. Ortmann, D. and Vallier, L. (2017) Variability of Human Pluripotent Stem Cell Lines. Current Opinion in Genetics \& Development, 46, 179-185. https://doi.org/10.1016/j.gde.2017.07.004

6. Krause, D.S., Scadden, D.T. and Preffer, F.I. (2013) The Hematopoietic Stem Cell Niche-Home for Friend and Foe? Cytometry. Part B, Clinical Cytometry, 84, 7-20. https://doi.org/10.1002/cyto.b.21066

7. Morrison, S.J. and Scadden, D.T. (2014) The Bone Marrow Niche for Haematopoietic Stem Cells. Nature, 505 327-334. https://doi.org/10.1038/nature12984

8. Choi, J.S., Mahadik, B.P. and Harley, B.A. (2015) Engineering the Hematopoietic Stem Cell Niche: Frontiers in Biomaterial Science. Biotechnology Journal, 10, 1529-1545. https://doi.org/10.1002/biot.201400758

9. Turksen, K. (2016) Bioreactors in Stem Cell Biology. Methods Mol Biol, 1502. Humana Press.

10. Dos Santos, F., Campbell, A., Fernandes-Platzgummer, A., et al. (2014) A Xenogeneic-Free Bioreactor System for the Clinical-Scale Expansion of Human Mesenchymal Stem/Stromal Cells. Biotechnology and Bioengineering, 111, 1116-1127. https://doi.org/10.1002/bit.25187

11. Collins, P.C., Miller, W.M. and Papoutsakis, E.T. (1998) Stirred Culture of Peripheral and Cord Blood Hematopoietic Cells Offers Advantages over Traditional Static Systems for Clinically Relevant Applications. Biotechnology and Bioengineering, 59, 534-543. https://doi.org/10.1002/(SICI)1097-0290(19980905)59:5<534::AID-BIT2>3.0.CO;2-B

12. He, W., Ye, L., Li, S., et al. (2012) Stirred Suspension Culture Improves Embryoid Body Formation and Cardiogenic Differentiation of Genetically Modified Embryonic Stem Cells. Biological and Pharmaceutical Bulletin, 35, 308-316. https://doi.org/10.1248/bpb.35.308

13. Tsai, A.C., Liu, Y., Yuan, X., et al. (2017) Aggregation Kinetics of Human Mesenchymal Stem Cells under Wave Motion. Biotechnology Journal, 12. https://doi.org/10.1002/biot.201600448

14. Singh, V. (1999) Disposable Bioreactor for Cell Culture Using Wave-Induced Agitation. Cytotechnology, 30, 149-158. https://doi.org/10.1023/A:1008025016272

15. Begley, C.M. and Kleis, S.J. (2000) The Fluid Dynamic and Shear Environment in the NASA/JSC Rotating-Wall Perfused-Vessel Bioreactor. Biotechnology and Bioengineering, 70, 32-40. https://doi.org/10.1002/1097-0290(20001005)70:1<32::AID-BIT5>3.0.CO;2-V

16. Liu, Y., Liu, T., Fan, X., et al. (2006) Ex Vivo Expansion of Hematopoietic Stem Cells Derived from Umbilical Cord Blood in Rotating Wall Vessel. Journal of Biotechnology, 124, 592-601. https://doi.org/10.1016/j.jbiotec.2006.01.020

17. De Napoli, I.E., Scaglione, S., Giannoni, P., et al. (2011) Mesenchymal Stem Cell Culture in Convection-Enhanced Hollow Fibre Membrane Bioreactors for Bone Tiss. Fuel and Energy Abstracts.

18. Gerlach, J.C., Lin, Y.C., Brayfield, C.A., et al. (2012) Adipogenesis of Human Adipose-Derived Stem Cells within Three-Dimensional Hollow Fiber-Based Bioreactors. Tissue Engineering, Part C Methods, 18, 54-61.

https://doi.org/10.1089/ten.tec.2011.0216

19. Amit, M., Chebath, J., Margulets, V., et al. (2010) Suspension Culture of Undifferentiated Human Embryonic and Induced Pluripotent Stem Cells. Stem Cell Reviews and Reports, 6, 248-259.

https://doi.org/10.1007/s12015-010-9149-y

20. Elanzew, A., Sommer, A., Pusch-Klein, A., et al. (2015) A Reproducible and Versatile System for the Dynamic Expansion of Human Pluripotent Stem Cells in Suspension. Biotechnology Journal, 10, 1589-1599. https://doi.org/10.1002/biot.201400757 
21. Khosrowshahi, Y.B., Khoshfetrat, A.B., Abolghasemi, Z. and Shams Asenjan, K. (2015) Performance Evaluation of a Proliferation Chamber with External Stirred Conditioning Tank for Expansion of a Suspendable Stem Cell Model. Process Biochemistry, 50, 1110-1118. https://doi.org/10.1016/j.procbio.2015.04.009

22. King, J.A. and Miller, W.M. (2007) Bioreactor Development for Stem Cell Expansion and Controlled Differentiation. Current Opinion in Chemical Biology, 11, 394-398. https://doi.org/10.1016/j.cbpa.2007.05.034

23. Yeatts, A.B. and Fisher, J.P. (2011) Bone Tissue Engineering Bioreactors: Dynamic Culture and the Influence of Shear Stress. Bone, 48, 171-181. https://doi.org/10.1016/j.bone.2010.09.138

24. Plett, P.A., Abonour, R., Frankovitz, S.M. and Orschell, C.M. (2004) Impact of Modeled Microgravity on Migration, Differentiation, and Cell Cycle Control of Primitive Human Hematopoietic Progenitor Cells. Experimental Hematology, 32, 773-781. https://doi.org/10.1016/j.exphem.2004.03.014

25. Schwarz, R.P., Goodwin, T.J. and Wolf, D.A. (1992) Cell Culture for Three-Dimensional Modeling in Rotating-Wall Vessels: An Application of Simulated Microgravity. Journal of Tissue Culture Methods, 14, 51-57. https://doi.org/10.1007/BF01404744

26. Nold, P., Brendel, C., Neubauer, A., et al. (2013) Good Manufacturing Practice-Compliant Animal-Free Expansion of Human Bone Marrow Derived Mesenchymal Stroma Cells in a Closed Hollow-Fiber-Based Bioreactor. Biochemical and Biophysical Research Communications, 430, 325-330.

https://doi.org/10.1016/j.bbrc.2012.11.001

27. Hanley, P.J., Mei, Z., Durett, A.G., et al. (2014) Efficient Manufacturing of Therapeutic Mesenchymal Stromal Cells with the Use of the Quantum Cell Expansion System. Cytotherapy, 16, 1048-1058.

https://doi.org/10.1016/j.jcyt.2014.01.417

28. Lambrechts, T., Papantoniou, I., Rice, B., et al. (2016) Large-Scale Progenitor Cell Expansion for Multiple Donors in A Monitored Hollow Fibre Bioreactor. Cytotherapy, 18, 1219-1233.

https://doi.org/10.1016/j.jcyt.2016.05.013

29. Lambrechts, T., Papantoniou, I., Viazzi, S., et al. (2016) Evaluation of a Monitored Multiplate Bioreactor for Large-Scale Expansion of Human Periosteum Derived Stem Cells for Bone Tissue Engineering Applications. Biochemical Engineering Journal, 108, 58-68. https://doi.org/10.1016/j.bej.2015.07.015

30. Bratt-Leal, A.M., Kepple, K.L., Carpenedo, R.L., et al. (2011) Magnetic Manipulation and Spatial Patterning of Multi-Cellular Stem Cell Aggregates. Integrative Biology (Camb), 3, 1224-1232.

https://doi.org/10.1039/c1ib00064k

31. Liu, H., Lin, J. and Roy, K. (2006) Effect of 3D Scaffold and Dynamic Culture Condition on the Global Gene Expression Profile of Mouse Embryonic Stem Cells. Biomaterials, 27, 5978-5989.

https://doi.org/10.1016/j.biomaterials.2006.05.053

32. Meinel, L., Karageorgiou, V., Fajardo, R., et al. (2004) Bone Tissue Engineering Using Human Mesenchymal Stem Cells: Effects of Scaffold Material and Medium Flow. Annals of Biomedical Engineering, 32, 112-122. https://doi.org/10.1023/B:ABME.0000007796.48329.b4

33. Hosseinkhani, H., Azzam, T., Kobayashi, H., et al. (2006) Combination of 3D Tissue Engineered Scaffold and Non-Viral Gene Carrier Enhance In Vitro DNA Expression of Mesenchymal Stem Cells. Biomaterials, 27, 4269-4278. https://doi.org/10.1016/j.biomaterials.2006.02.033

34. Ertl, P., Sticker, D., Charwat, V., et al. (2014) Lab-on-a-Chip Technologies for Stem Cell Analysis. Trends in Biotechnology, 32, 245-253. https://doi.org/10.1016/j.tibtech.2014.03.004

35. Gupta, K., Kim, D.-H., Ellison, D., et al. (2010) Lab-on-a-Chip Devices as an Emerging Platform for Stem Cell Biology. Lab on a Chip, 10, 2019-2031. https://doi.org/10.1039/c004689b

36. Faley, S.L., Copland, M., Wlodkowic, D., et al. (2009) Microfluidic Single Cell Arrays to Interrogate Signalling 
Dynamics of Individual, Patient-Derived Hematopoietic Stem Cells. Lab Chip, 9, 2659-2664. https://doi.org/10.1039/b902083g

37. Kamei, K., Guo, S., Yu, Z.T., et al. (2009) An Integrated Microfluidic Culture Device for Quantitative Analysis of Human Embryonic Stem Cells. Lab Chip, 9, 555-563. https://doi.org/10.1039/B809105F

38. Villa-Diaz, L.G., Torisawa, Y.S., Uchida, T., et al. (2009) Microfluidic Culture of Single Human Embryonic Stem Cell Colonies. Lab Chip, 9, 1749-1755. https://doi.org/10.1039/b820380f

39. Kim, C., Lee, K.S., Bang, J.H., et al. (2011) 3-Dimensional Cell Culture for On-Chip Differentiation of Stem Cells in Embryoid Body. Lab Chip, 11, 874-882. https://doi.org/10.1039/c0lc00516a

40. Zhang, Y.X., Wang, Z.Y., Farhangfar, F. and Zimmerman, M. (2017) In Vitro Evaluation of Anticancer Drugs with Kinetic and Static Alternating Cell Culture System. Journal of Cancer Therapy, 08, 845-859. https://doi.org/10.4236/jct.2017.89074

41. Zhang, Y. and Wang, Y. (2016) Cancer Specific CTL Expansion with ZYX Bioreactor. Journal of Clinical \& CelIular Immunology, 7, 398.

42. Mohyeldin, A., Garzon-Muvdi, T. and Quinones-Hinojosa, A. (2010) Oxygen in Stem Cell Biology: A Critical Component of the Stem Cell Niche. Cell Stem Cell, 7, 150-161. https://doi.org/10.1016/j.stem.2010.07.007

43. Fotia, C., Massa, A., Boriani, F., et al. (2015) Hypoxia Enhances Proliferation and Stemness of Human Adipose-Derived Mesenchymal Stem Cells. Cytotechnology, 67, 1073-1084.

https://doi.org/10.1007/s10616-014-9731-2

44. Parmar, K., Mauch, P., Vergilio, J.A., et al. (2007) Distribution of Hematopoietic Stem Cells in the Bone Marrow According to Regional Hypoxia. Proceedings of the National Academy of Sciences USA, 104, 5431-5436. https://doi.org/10.1073/pnas.0701152104

45. Mantel, C.R., O'Leary, H.A., Chitteti, B.R., et al. (2015) Enhancing Hematopoietic Stem Cell Transplantation Efficacy by Mitigating Oxygen Shock. Cell, 161, 1553-1565. https://doi.org/10.1016/j.cell.2015.04.054

46. Grayson, W.L., Zhao, F., Bunnell, B. and Ma, T. (2007) Hypoxia Enhances Proliferation and Tissue Formation of Human Mesenchymal Stem Cells. Biochemical and Biophysical Research Communications, 358, 948-953. https://doi.org/10.1016/j.bbrc.2007.05.054

47. Dos Santos, F., Andrade, P.Z., Boura, J.S., et al. (2010) Ex Vivo Expansion of More Effective Cell Proliferation Kinetics and Metabolism under Hypoxia. Journal of Cellular Physiology, 223, 27-35.

48. Fernandes, A.M., Fernandes, T.G., Diogo, M.M., et al. (2007) Mouse Embryonic Stem Cell Expansion in a Microcarrier-Based Stirred Culture System. Journal of Biotechnology, 132, 227-236. https://doi.org/10.1016/j.jbiotec.2007.05.031

49. Sen, A., Kallos, M.S., Behie, L.A. (2002) Expansion of Mammalian Neural Stem Cells in Bioreactors: Effect of Power Input and Medium Viscosity. Brain Research. Developmental Brain Research, 134, 103-113. https://doi.org/10.1016/S0165-3806(01)00328-5

50. Fernandes-Platzgummer, A., Diogo, M.M., Baptista, R.P., et al. (2011) Scale-Up of Mouse Embryonic Stem Cell Expansion in Stirred Bioreactors. Biotechnology Progress, 27, 1421-1432. https://doi.org/10.1002/btpr.658

51. Heathman, T.R., Glyn, V.A., Picken, A., et al. (2015) Expansion, Harvest and Cryopreservation of Human Mesenchymal Stem Cells in a Serum-Free Microcarrier Process. Biotechnology and Bioengineering, 112, 1696-1707. https://doi.org/10.1002/bit.25582

52. Mizukami, A., Fernandes-Platzgummer, A., Carmelo, J.G., et al. (2016) Stirred Tank Bioreactor Culture Combined with Serum-/Xenogeneic-Free Culture Medium Enables an Efficient Expansion of Umbilical Cord-Derived Mesenchymal Stem/Stromal Cells. Biotechnology Journal, 11, 1048-1059.

https://doi.org/10.1002/biot.201500532 
53. Santos, F., Andrade, P.Z., Abecasis, M.M., et al. (2011) Toward a Clinical-Grade Expansion of Mesenchymal Stem Cells from Human Sources: A Microcarrier-Based Culture System under Xeno-Free Conditions. Tissue Engineering, Part C Methods, 17, 1201-1210. https://doi.org/10.1089/ten.tec.2011.0255

54. Csaszar, E., Kirouac, D.C., Yu, M., et al. (2012) Rapid Expansion of Human Hematopoietic Stem Cells by Automated Control of Inhibitory Feedback Signaling. Cell Stem Cell, 10, 218-229. https://doi.org/10.1016/j.stem.2012.01.003

55. Bardy, J., Chen, A.K., Lim, Y.M., et al. (2013) Microcarrier Suspension Cultures for High-Density Expansion and Differentiation of Human Pluripotent Stem Cells to Neural Progenitor Cells. Tissue Engineering, Part C Methods, 19, 166-180. https://doi.org/10.1089/ten.tec.2012.0146

56. Frauenschuh, S., Reichmann, E., Ibold, Y., et al. (2007) A Microcarrier-Based Cultivation System for Expansion of Primary Mesenchymal Stem Cells. Biotechnology Progress, 23, 187-193. https://doi.org/10.1021/bp060155w

57. Chen, A.K., Chen, X., Choo, A.B., et al. (2010) Expansion of Human Embryonic Stem Cells on Cellulose Microcarriers. Current Protocols in Stem Cell Biology, Chapter 1, Unit 1C 11.

58. Leung, H.W., Chen, A., Choo, A.B.H., Reuveny, S. and Oh, S.K.W. (2010) Agitation Can Induce Differentiation of Human Pluripotent Stem Cells in Microcarrier Cultures. Tissue Engineering Part C: Methods, 17, 165-172. https://doi.org/10.1089/ten.tec.2010.0320

59. Park, Y., Chen, Y., Ordovas, L. and Verfaillie, C.M. (2014) Hepatic Differentiation of Human Embryonic Stem Cells on Microcarriers. Journal of Biotechnology, 174, 39-48. https://doi.org/10.1016/j.jbiotec.2014.01.025

60. dos Santos, F.F., Andrade, P.Z., da Silva, C.L. and Cabral, J.M. (2013) Bioreactor Design for Clinical-Grade Expansion of Stem Cells. Biotechnology Journal, 8, 644-654. https://doi.org/10.1002/biot.201200373

61. Zhang, Y.X., et al. (2016) Cancer Specific CTL Expansion with ZYX Bioreactor. Journal of Clinical \& Cellular Immunology, 7, 398. https://doi.org/10.4172/2155-9899.1000398

62. Fan, Y., Zhang, F. and Tzanakakis, E.S. (2017) Engineering Xeno-Free Microcarriers with Recombinant Vitronectin, Albumin and UV Irradiation for Human Pluripotent Stem Cell Bioprocessing. ACS Biomaterials Science \& Engineering, 3, 1510-1518. https://doi.org/10.1021/acsbiomaterials.6b00253

63. Lin, C.-Y., Huang, C.-H., Wu, Y.-K., et al. (2014) Maintenance of Human Adipose Derived Stem Cell (hASC) Differentiation Capabilities Using a 3D Culture. Biotechnology Letters, 36, 1529-1537. https://doi.org/10.1007/s10529-014-1500-y

64. Yongxin Zhang FF, Wang, Y., et al. (2017) Assessment of Differentiation States of Hematopoietic Stem Cells Following In Vitro Culture Using Side and Forward Scatter of Flow Cytometry. World Journal of Research and Review, 5, 75-83.

65. Sutherland, D.R., Anderson, L., Keeney, M., et al. (1996) The ISHAGE Guidelines for CD34+ Cell Determination by Flow Cytometry. International Society of Hematotherapy and Graft Engineering. Journal of Hematotherapy, 5, 213-226. https://doi.org/10.1089/scd.1.1996.5.213 